Neil Hooley

\title{
38. Participation and the Question of Knowledge
}

It is quite amazing how a piece of writing is never really finished, how the most carefully crafted and thoughtful essay has obvious gaps and weaknesses when returned to at a later stage. Readers of an academic manuscript may expect more than text that masks an underlying uncertainty. Even so, I find myself wanting to increasingly express my discursive and incomplete thinking as I range across difficult issues in teacher education, encompassing as they do the full gamut of philosophical, epistemological and political problems that society creates.

As I sit back and view the sunset, I am now comfortable with the notion that the best we can do is work through difficult issues with others in cycles of investigation and reflection and attempt to enhance our mutual understanding if we can. It is frustrating to try to find the appropriate words to describe the process and the position reached, while at the same time realising that whatever the choice, the reader must interpret an understanding that is inadequately expressed. Even the esteemed Oxford mathematician Roger Penrose in his latest work (Penrose, 2004, p. 1028) has cast doubt on the quest within physics for a 'theory of everything' and whether it is possible 'to find 'reality' within the Platonic world of mathematical ideals.' If mathematics cannot provide the certainty we seek for explanation and guidance, then my meanderings will never reach a conclusion.

My intention below is to sketch some of these meanderings, the educational and cultural questions that have dominated my life for many years and which I have struggled to resolve. I shall make some comment on research and knowledge and the idea of narrative as a useful construct for personal change and understanding. My thoughts have been strongly influenced over recent times by such matters within the context of Australian Indigenous learning. There is no more difficult or important question in Australian education, indeed within Australia itself and until such time as reconciliation between the Indigenous and non-Indigenous peoples is achieved, we shall all be diminished. We need to seek anchor points within the recognised literature to enable this to occur within education and to provide models of learning that are accessible to all regardless of biography. A critical relationship with knowledge seems to me to be the key in allowing some intellectual purchase on this problem and a willingness to think any thought, to scale any mountain that obstructs our path. But to do so, we need to reach some type of consensus on knowledge itself, to agree on what we are talking about, at least in the short term.

A philosophical framework for thinking about knowledge production may consider human ideas and understanding as emerging from empirical, hermeneutic, or critical investigations. The first approach sees truth or trustworthiness residing in various forms of experiment and measurement, the second moves beyond mere observation and requires human interpretation of whatever data is at hand and the third, application of a world 
view that examines personal bias and prejudice, power relations and social purpose. The shift from a more positivist to a more emancipatory frame of knowledge has been an ongoing process throughout the Enlightenment period and is found across the academic disciplines to greater or lesser extents. To this we might add the notion of participatory research that sees understanding arising from communities of learning and research which have an explicit socio-cultural perspective and which engage in the robust contestation of ideas, principles and values as reflective cycles of investigation unfold.

Under these circumstances of critical perspective and participation, knowledge production is non-neutral and generalisable but must always be refined and validated through practice and participation. Experience within an Australian Bachelor of Education program for example and some informal reflections reported below indicates that at least to some extent, a critical participation can form the basis of both teaching and research where staff and students constantly undertake knowledge production and critique over extended periods of time. A central aspect of participatory research is the written documentation of experience and reflection on how the research process itself challenges personal ideas and practice. In this way, research outcomes involve not only new personal knowledge but changes to the researchers themselves which, in the end, is the basis of enduring change. For the author, the drafting of the following reflections on naturalistic methodology as 'understanding in progress' has been another step in that journey.

\section{Thinking about teaching and research}

Research as an investigation into knowledge and undertaken from a critical point of view will begin with the question, what is the point of conducting this research if the researchers remain unchanged by the experience? That is, have the researchers learnt nothing about themselves and hold exactly the same views and understandings as they did at the beginning of the project? How will this make for a better world, how will the status quo be altered? Of course, learning, outcomes, or findings are relative terms and can be seen as technical and constraining or critical and liberating. It may be that the work itself is purely or mainly empirical, has scope for interpretation, or can establish a framework of practice and reflection that is deeply challenging of personal values and beliefs. This is the classic contradiction of modern science where research can be conducted for its own sake regardless of the purposes to which the new knowledge can be put. Research of this type is seen as disconnected from our prejudice, bias and irrationality. But research of this type is also a fantasy.

In discussing a reconceptualisation of knowledge within a context of modern and postmodern critiques of curriculum for instance, Moore and Young (2001, p.459) conclude with a description of knowledge as the 'historically located collective achievement of human creativity.' While positioning knowledge as an artefact of social action, this concept leaves open the process by which such an achievement actually takes place. For example, two epistemological frameworks are possible when considering how humans go about creating or discovering new knowledge (Chalmers, 1996; Edelman and Tononi, 2000). Both are models to assist our understanding of the human condition at this 
time. In the first approach, a mind-body duality exists where the mind is separate from both body and brain and there is an absolute distinction between material and mental processes. A second view suggests that mind emerges from the physical properties of matter as encountered in the body, particularly as a certain threshold of complexity is exceeded and new properties such as consciousness are formed. In a broader sense, both models draw sharp distinctions between a theological and scientific explanation of the universe. If mind is 'supra-material' then it owes allegiance to a superior being, whereas if mind is mere matter its existence is simply another feature of the universe and not extraordinary in any way. On this latter basis, the vast reaches of the universe could quite easily be characterised by life and consciousness.

The complexity model (Johnson, 2001) needs to be able to show or at least hypothesise how ideas, values, emotions and morality can arise from matter. This is an extremely difficult task for both philosophy and science. It will involve theorised connections between experience and nature, between action and thought and between feedback, reflection and communication. Such investigations will draw upon the insights of information theory and cybernetics, neurobiology, cosmology and philosophy. An important component of this idea of 'emergence' is a possible explanation of human consciousness, that is how humans are aware of their own histories. This project is sometimes referred to as the 'hard problem' of philosophy (Chalmers, 2002, p. 92) and perhaps the last on which consensus may be reached. For researchers and educators it may be possible to design programs based on certain perspectives of each model without resolving each particular detail. What is important here is that each model is not ignored and that the framework guiding human action of whatever kind is acknowledged, evaluated and changed as human activity and interaction proceeds.

As well as consciousness, proponents of either model must confront questions of neutrality, subjectivity, objectivity and validation and indeed reach agreement on the philosophical baseline before the actual work of projects unfold (Anderson and Herr, 1998; Coulter, 2002, Eisner, 2002). If thought and perspective arise from material and the experience of material occurs in both nature and society, then all ideas develop within a socio-cultural context and cannot be neutral. As humans make decisions and judgements on all experience the results of which ultimately find their way to the brain in the form of electrical impulses, then it follows that the connections between action, experience, nature, society cannot be severed, one is always a part of the other. Rather than an independent mind fashioning human cognition in a manner disconnected from experience, the human organism comes to know through not only connecting with experience, but becoming experience. Rather than the abyss, a movement between subjectivity and objectivity occurs where the knowing subject attempts to establish a relationship with the object to be known with understanding being a function of movement between the two. Knowledge is true when humans agree that it is true at least until new experience casts doubt on the outcomes of action.

Educational research is generally not conducted from an acknowledged and defensible philosophical position. According to St Pierre (2002, p. 26), 'Much educational research, in fact, does not even acknowledge its epistemological grounding, much less take into 
account the limits of that epistemology and its methodology, in the production of knowledge'. Issues regarding methodology as distinct from method can be assumed. For research in the social sciences particularly when working with local communities where democratic and respectful arrangements are crucial, where the knowledge, wisdom and understandings of groups of people constitute the basis of the research, ignoring such matters will mean that the direction of the research will be poorly defined, problems are more difficult to resolve and interpretations become more disruptive than cohesive.

\section{Viewing knowledge with a critical lens}

The notion of being critical and of developing critique has a long history in both the social and physical fields throughout the modern era (Young, 1990). It has in fact been an important characteristic of the modern era. Galileo, Kepler and Copernicus dared to think about the place of the Earth in the universe against the established wisdom of the church. Marx, Darwin and Freud put forward revolutionary ideas about the human condition. The pragmatic philosophers placed emphasis on practice, enquiry and the individual interest. Bohr challenged Einstein and Newton in regards quantum mechanics, theorists of the Frankfurt School developed a cultural criticism of capitalism, Freire took up the issue of illiteracy and exploitation of the Brazilian peasants. More recently, the postmodern view has identified a range of social features for ideology critique. Researchers always have a very clear choice therefore of seeing their work as being subservient to or being critical of the current socio-economic and scientific paradigm. They do not have a choice of their work being neutral.

A critical theorist is interested in both theory and practice, indeed the theorising of practice and may see each as being the same as the other, that is all phenomena are constituted by a practice/theory unity or dialectic. The research task is to consider the theory that guides practice and the practice that informs theory, to untangle the ideas that are behind every act so that substantial change and improvement are possible. It also means that the participants in the research process must also consider the impact that the research has on them, their views and predilections. As Shacklock and Smyth (1998, p. 4) suggest, 'The intent is to engage in a constant questioning and building up of theory and interpretations through repeated ongoing analysis until a coherent alternative reconstruction of the account is created.' The notion of conversation between ideas and data is important here. If research and knowledge are not neutral but arise from the ideological determinants of society then a dialectical process will also exist between the socio-political views of participants and the emergent understandings. These issues need to be built into the research design, to ensure that personal, political and epistemological perspectives are known so that their influence on the research as it unfolds can be dealt with as necessary. The changes that researchers themselves undergo should be explicitly discussed throughout the project particularly as they impact on the observations, interpretations, analyses and hypotheses as they occur.

For research to take up emancipatory interest, it must be undertaken by groups of researchers rather than individuals, involve explicit linkages with major social and political events, contain the services of a critical friend for advice and experience that the 
group may not have and expressly locate itself within the critical tradition as mentioned above. This means the full democratic participation of all participants as equals with open discussions regarding ideological positions from the beginning of the project. Tricoglus (2001) takes up similar issues in discussing a tentative protocol for a practitioner critical ethnography and identifies issues such as establishing the purpose and theoretical basis of research, knowing the worldview and context of participants, knowing the data and yourself. In summary, participation is quite a different concept to that of collaboration and needs to include the following features:

- research and knowledge seen as historical and ideological

- context of research dependent on socio-economic and cultural conditions

- research groups seek to transform reality and basis of oppression

- draws upon the explicit understandings and experiences of participants

- unity of practice and theory, ideas and action, method of social praxis

- new knowledge emerges from reflection on broad experiential base

- connects with other social groupings and colleagues for advice, challenge

- validity of knowledge arises from application, communication, negotiation

- transforms consciousness from technical to critical

- lifelong perspective, involving shorter and longer cycles of investigation.

\section{Bourdieu and the field of educational research}

The above discussion reminds us of the work of the French sociologist Pierre Bourdieu, his notions of field and habitus and particularly the relation between them. He describes the 'proper object of social science' (Bourdieu, 1992, pp. 126 - 127) as the 'relation between two realisations of historical action' in the following terms:

It is the double and obscure relations between habitus ie the durable and transposable systems of schemata of perception, appreciation and action that result from the institution of the social in the body (or in biological individuals) and fields ie systems of objective relations which are the product of the institution of the social in things or in mechanisms that have the quasi reality of physical objects; and of course, of everything that is born of this relation, that is, social practices and representations, or fields as they as they present themselves in the form of realities perceived and appreciated.

Bourdieu is clear that social science must direct its attention not to the individual but to the field, its properties and positions, meaning that the focus of research projects is to identify, clarify, characterise those features that impact on social action and which can therefore be changed to influence different outcomes. For educational research, the field would comprise in part ideological approaches towards economic and cultural development, conflict between philosophies of modernity and post modernity, tensions between ontological and epistemological frames of knowledge production, viewpoints of social class, gender, ethnicity. This is clearly complicated work. An analysis of the field would consider the power relations that exist between positions such as the status of research teams when competing for funding from agencies, methodologies that are more qualitative or more quantitative, the question of 'voice' amongst participants, national 
priorities that emphasise empirical rather than interpretive outcomes. These are seen in relation to the habitus of researchers, their backgrounds, perceptions, culture, worldview and morality, the range of capitals that constitute their very existence.

The significance of Bourdieu's insights for educational research is that the researchers are dislodged from their insulated capsules of neutrality and, regardless of the specific interest of their projects, must confront the realities of interlocking ideology, context and prejudice. These reside in the habitus and need to be exposed for scrutiny as relations and essentials of the research process. Conceptualising educational research as a field for social action, where the ideas and practices of practitioners is a field of epistemological contestation and understanding for all participants will shake dispositions, values, stereotypes to their very foundations.

\section{Indigenous knowing: thinking across worlds}

Ruminating on these types of ideas, that is, different approaches to knowledge and learning, the place of participation and how human consciousness actually comes into being for understanding, are central considerations for reconciliation between the Indigenous and non-Indigenous peoples of Australia. Following settlement of Australia by the British in the 1770s and 80s, the overall health and well being of the Indigenous population has steadily declined. The Indigenous people comprise approximately two percent of the overall population and mainly live in cities and towns down the east coast as do most other Australians. A small proportion live in tiny Indigenous communities in remote areas of the country. With the large-scale dispossession of their traditional lands and the resultant destruction of customs and languages, the struggle of the Indigenous peoples has been to live within two worlds while attempting to maintain their own culture and knowledge systems. In many cases, this has proven to be an almost impossible task.

Of particular concern within Australia of course, is how to provide a curriculum in regular schools that is inclusive of Indigenous ways of knowing. Primary schools are better placed in this regard with their integrated approaches, but the segmented nature of the secondary curriculum where knowledge is disconnected amongst itself and from the broader society in which it is located, is a significant factor in the high drop out rate that occurs. Contradictions that exist between the school organization and curriculum and the way that children learn within their communities, become too much to bear. Insufficient attention is given to Indigenous learning styles (Hughes and Moore, 1997) and the notions of family, community, sharing and co-operation that permeate Indigenous life around the world. Learning from the land and natural environment is another important principle that is overlooked, even though it provides obvious linkages with western science and the school curriculum. Ma Rhea (2004, p. 9) makes the telling point that the incorporation of empirical Indigenous knowledge into western education 'raises the important question of what theoretical and methodological approaches should be adopted' to ensure that such knowledge is treated with respect and is not distorted or misrepresented. These are huge issues that may not have been resolved by dominant settler societies in very many places around the world. 
In attempting to grapple with these matters, I have proposed the idea of 'two-way enquiry learning' (Hooley, 2002) that brings together the general approach to inquiry as advocated by Dewey (1963) and 'two-way learning' in the Australian Indigenous context as suggested by Harris (1990). Participation is a key feature of this approach, democratic participation by Indigenous people in their own education and direct participation in the generation and refinement of knowledge over time. If this is to occur, then new forms of curriculum need to be recognised that in fact challenge the authority of existing structures in the way that knowledge is conceived and privileged. Given the importance of oral, artistic and ritual communication in Indigenous communities, these new curriculum forms will need to take due account of human expression in all its forms, rather than the European insistence of writing only. This also means that due respect and recognition for the Indigenous peoples of Australia within regular schools and university programs through more open, democratic and communicative mechanisms should improve the learning for all students whatever their socio-cultural background.

\section{Constructing narrative as research}

For researchers concerned with democratic knowing (Reason and Torbert, 2001; Sanderson and Allard, 2001; Schultz, 2001), particularly when working with communities and practitioners, methodologies must be employed that respect and recognise local practices, knowledges and cultures so that findings are grounded in experience and socio-cultural intent. Approaches that utilise narrative are congruent with the philosophy of participatory action research and enable understandings to be fluidly constructed over time as a project unfolds. Polkinghorne (1988, p. 161) has identified two types of narrative that embody these functions. The first or descriptive aims to outline narratives that already exist and provide the 'means for ordering and making temporal events meaningful.' The second or explanatory 'ties together and orders events so as to make apparent the way they 'caused' the happening under investigation'. In drawing parallels between the law and qualitative research, Donlevy (2003) notes that affidavits, opening addresses by lawyers and the summary by judges are narratives accepted by the court as reliable accounts. Juries are involved in a process very similar to qualitative research where recognised procedures are adopted and where narratives, stories and evidence are given from expert witnesses, direct participants and critical friends resulting in outcomes that are taken to be trustworthy.

Within the concept and practice of narrative (Clandinin and Connelly, 2000; Bullough and Pinnegar, 2001), democratic participation is an essential element in the production of new knowledge where greater definition can be given to human understanding of social and physical existence. There is a direct connection and movement between the human capacity for judgement, decision making, ideas and values on the one hand and the rich substrate of experience and reflection on the other. Ultimately, this process leads to a critical consciousness, the capacity of humans to not only be aware of and think about their own biographies, but to provide critique of their own cognition and activity from a moral and political viewpoint. 
A holistic and integrated approach to research and knowledge, indeed to life itself, enables participants to reflect upon their experience and to engage a process that challenges personal ideas, values and practices. The following two e-mail messages written by myself as lecturer indicate the role that writing can play in assisting reflective thought and the possibility for critical consciousness. The first describes an incident from an Australian Bachelor of Education class for final year pre-service teachers:

'We could be doing what Freire said!' This comment from a student blew me away, my excitement almost uncontained. It came towards the end of our Year 4 case conference when we were discussing the data as a whole. As the beginning teachers had noted the changes in their own practice and thinking amply shown in the cases, I had taken the opportunity to make a few points about the series of transitions that I see happening throughout the BEd. These are about seven in all I estimate, ranging from a pre-university to university transition and then 3/4 years later, the university to becoming professional transition ie from being a university student to a disciplined practitioner. While the latter is to be expected if the course structure is accurate and enabling, a final transition that is not required of graduates is movement towards that of critical consciousness as described by Freire where our thinking and reflection becomes that of personal, organisational and structural critique for social change, perhaps recognition of a structure/agency duality. The case conference had thrown up comments like 'taking ourselves out of ourselves' an insight that had certainly not been there earlier in the year, although the notion of 'working hypothesis' introduced by myself as critical friend, had been accepted readily as a means for the group to think about and investigate itself. The remark about Freire was hugely significant and spontaneous arising from our consideration of the case data and seemed to me to indicate the growing professional understanding that was present and emerging; is great leaps going too far? To witness new thresholds and ideas condense from the vapours of practical experience is evidence of transformation from one state to another.

(e-mail communication, 18 October 2002)

This is a personal reflection on my part. The comment regarding Freire (Freire, 1998; Glass, 2001) may have been completely innocent, co-incidental. But I suspect not. The structure of the four-year Bachelor of Education has been carefully constructed to enable learning by doing, the establishment of partnership teams between the university and schools where team members are immersed in the difficult experience of professional practice and from which they must make their own interpretations. If the structure is reasonably supportive of participant action and reflection, then practice will be underpinned by theory when the need arises, what has been read and discussed will become applicable to guide understanding and new insights will emerge as the mass of complex experience is reached. Hopefully the critical friend as participant will be attuned to such incidents as they occur.

For a researcher concerned with the production of new knowledge from a context of maximum participation, the separation between life and research is blurred. All that 
occurs contributes to the energy of the brain and its transformation into thought no matter how confused. Conversations with students in the corridor, meetings with teachers at a school, interactions between students and teachers, political events occurring worldwide, a beautiful sunset or dust storm, decisions regarding personal finances, all cannot be excluded from the experiential base, in fact, they form the experiential base. All of these are also generated from the culture within which the research groups finds itself and from which its collective thinking cannot be disconnected. Freire had been discussed to varying extents for example at different times over the previous four years and perhaps it had taken this long for the complex structures of the brain to generate a new thought similar to a 'big bang' process.

The second message concerns another personal reflection on the question of consciousness as noted above and stimulated by the experience of class discussions within the Bachelor of Education program and personal reading:

Given the exciting developments of modern science at the time, it is understandable that both Dewey and Freud sought scientific explanation for a theory of mind and human understanding. They differed markedly however on Freud's unconscious that creates its own reality and imperatives and Dewey's consciousness that emerges from experience and links mind, nature and culture. Dewey's view is not that far removed from what is now called complexity theory when he suggests that mind is a function of matter and that consciousness enables humans to learn from experience, to make decisions and judgements and to develop morality. In part, this is the debate between theism and materialism. One hundred years later, it would be interesting to have his assessment of the struggle that cosmologists let alone philosophers now have of uniting matter, energy, information, complexity, consciousness.

Considerations such as these inform undergraduate programs particularly those involving education and knowledge workers such as teachers. A broadly philosophical approach around mathematics and information and communication technologies for example, would refocus learning around fundamental questions of epistemology rather than the mere imparting of skill in classrooms that is of little benefit to anyone. Adopting Dewey's views as a guide would emphasise the child's theoretical construction of mathematical ideas and a practical reconstruction of their meaning, rather than a simplistic transmission of universal truths that are said to already exist. The problem of entropy made it difficult for both Freud and Dewey to explain how energy is converted into thought, but today's theory of complexity should work in favour of the curriculum designer. To integrate mathematics and ICT into a philosophical investigation of knowledge begins to break open the intellectual straitjacket imposed on children in schools and reconfigures teacher education programs similarly.

Dewey's theory of mind in the era of Darwin, was evolutionary. He needed to be able to show how the brain was able to convert perceptions into judgements 
with aesthetic and moral value and to go beyond the immediate in space and time. Freud had also believed the connection between psychoanalysis and biology but it was difficult to prove. He placed importance on an emotional past, entrapment in a repressed and intellectual morass, whereas Dewey saw humans drawing on the experience of the past to create a new and reconstructed future. As mentioned previously, the models of cosmology showing how the universe might work, also inform our understanding of mind, if the universe develops and changes in response to the principles of its physical components, then so too does mind. In the end, there is only matter. Structures of formal programs need to locate themselves within the great narratives of the modern era such as these and not ignore the implications.

(e-mail communication 2 November 2002)

Why is the question of consciousness of such importance for the writer, for myself? Why does critical consciousness appear to offer explanation or a way forward when events are witnessed or experienced? From the theory of complexity, it would be argued that the writer's cognitive structures have been established from a working class culture of poverty and factory work, growing up in a coastal and rural environment, intense experience of opposition to war and teaching and teacher union activity. It is not clear as to why this experience has been seen as important to communicate in writing for many years, except a family history where reading and writing particularly of poetry featured, a classical situation of working class literacy. As a broader reading of academic and political texts ensued, the connections between social life and the need to act in a systematic way against exploitation and oppression became more acute. That is, as the connections between practice and theory became more obvious. This lived experience has become dominant and therefore has been transferred to the field of educational research. A person with a more middle class background would have a more middle class approach to research, where their life can be abstracted from research projects and knowledge itself, where 'objectivity' and 'neutrality' can be maintained, if not vigorously defended.

The work of left wing and critical political theorists and of the pragmatic philosophers have combined to make sense of what has been observed and experienced. There is of course under this approach a limit to how far humans can develop a critique of themselves. If consciousness is a function of matter then it is impossible to be entirely critical of events and personal action and views, we are in fact, trapped by our own constitution. How conscious can we become of our own consciousness and therefore of our own failings, inadequacies and limitations? The writing contained in this paper and the examples of informal yet reflective communication for comment and criticism, show some attempt at making experience and views public as they occur, little regard for correctness and a determination to engage a holistic and socio-cultural approach to research and knowledge.

Unending cycles of life

Where do reflections or musing of this type lead, what are the practical outcomes, what is the place of exploratory writing, is there really a dialectic between knowing and doing? 
As an example of such issues, Figure 38.1 below is an attempt to schematically depict the writer's current emergent thinkiing about the key features of education and of a tertiary education program in particular. It contains some terms that are beyond the scope of this chapter, but they have been included to give a more complete picture of the issues that need to be incorporated into a comprehensive model of educational practice. There is an attempt to bring together philosophical questions with those that embrace epistemological structure and educational organisation. They have been identified with the Australian Indigenous issues of reconciliation raised above as determining factors (see Hooley, 2002, for an expanded discussion).

Figure 38.1 about here

It is difficult to expose one's thoughts as an ongoing and tentative narrative to public scrutiny and criticism as part of a research process, much easier to argue aloofness and disconnection. To not only describe personal thoughts but attempt self-critique and a possible explanation as to their origin, to describe consciousness in action is almost impossible. The 'hard problem' in philosophy of explaining how humans experience the experience of consciousness will remain for some time. What appears possible and necessary is a rich and challenging experience on which systematic reflection can be undertaken with the subsequent generation of diverse views that can be enhanced or rejected. The artefacts of consciousness can be displayed and critiqued but not consciousness itself. This is a significant outcome of the process to this point, that the artefacts of consciousness expressed as ideas, strategies, schema, dilemmas, are central to any research process and are fashioned and refashioned by the acknowledged perspective of the participating group. Perspective becomes a technology working with the ideasaction dialectic. This process demands a life-long commitment to knowledge where all aspects of experience are connected to everything else and where truth although apparently consistent with reality today, may exhibit severe contradictions tomorrow.

\section{Afterword}

So where has our reflective and discursive narrative taken us, have issues been encountered that will benefit a more progressive teacher education? I have attempted to write reflectively in narrative form, exposing the thoughts that flow through my mind and which crowd in as an integrated whole. Many of the issues are too difficult for resolution at this time and will remain as background constructs while we struggle with daily practice. For me, Figure 38.1 is an important working diagram for teacher education, containing a schema for new perceptual and conceptual knowledge emerging as a result of experience, reflection and the writing of this chapter. It may not constitute new understandings for other readers and researchers.

The key idea that has occurred to me during the writing of this chapter is that of the connections between Indigenous knowing and critical consciousness and what we can learn from this for teaching and learning in schools. How do teachers work with this connection for example? Some theorising of mind and the place of participation has been included which raises serious implications for the design of tertiary education programs 
and teacher education specifically. It seems that teacher education programs that concentrate on technique alone and not the full scope of interrelationships contained in practice, will not impact on the world or teachers and children. As a further outcome of the writing itself, the final paragraph above indicates new perspectives for myself, with new references, ideas and avenues to be pursued; 'ideas in action' or 'understanding in progress' is the nature of the work involved. I invite all readers to participate in the confusing and uncertain journey of personal inquiry ahead.

\section{References}

Anderson, G. L. and Herr, K. (1998). The New Paradigm Wars: Is there room for rigorous practitioner research in schools and universities? Educational Researcher, 28 (5), 12-21.

Bourdieu, P. and Wacquant, J. D. (1992). An Invitation to Reflexive Sociology, The University of Chicago Press.

Bullough, R. V. and Pinnegar, S. (2001). Guidelines for Quality in Autobiographical Forms of Self-Study Research, Educational Researcher, 30(3), 13-21.

Chalmers, D. J. (2002). The Puzzle Of Conscious Experience, Scientific American, 12(1), 90-100.

Chalmers, D.J. (1996). The Conscious Mind: In Search of a Fundamental Theory, Oxford University Press.

Clandinin, D. J. and Connelly, F. M. (2000). Narrative Inquiry: Experience and Story in Qualitative Research, San Francisco: Jossey-Bass Publishers.

Coulter, D. (2002). What Counts As Action In Educational Action Research? Educational Action Research, 10(12), 189-206.

Dewey, J. (1963). Experience and Education, New York: Collier.

Donlevy, J. K. (2003). What Law Has To Teach The Social Researcher: Reliability and Validity in Qualitative Research, available at and accessed March 2003: http://www.ucalgary.ca/ distance/January_2003/pages/donlevy.html

Edelman, G. M. and Tononi, G. (2000). Consciousness: How Matter Becomes Imagination, Penguin Books.

Eisner, E. W. (2002). What Can Education Learn From The Arts About The Practice Of Education? Journal of Curriculum and Supervision, 18(1), 4-16.

Freire, P. (1998). Pedagogy of Freedom: Ethics, Democracy and Civic Courage, Rowman and Littlefield Publishers Inc. 
Glass, R. D. (2001). On Paulo Freire's Philosophy of Praxis and the Foundations of Liberation Education, Educational Researcher, 30(2), 15-25.

Hansen, D. T. (2002). Dewey’s Conception of an Environment for Teaching and Learning, Curriculum Inquiry, 32(3), 267-280.

Harris, S. (1990). Two-Way Aboriginal Schooling: Education and Cultural Survival, Canberra: Aboriginal Studies Press.

Hickman, L. A. and Alexander, T. M. (Eds) (1998). The Essential Dewey Vols I and 2, Indiana University Press.

Hooley, N. (2002). Two-Way enquiry Learning: Exploring the interface between Indigenous and non-Indigenous Knowing, Victoria University of Technology.

Hughes, P. and Moore, A. J. (1997). Aboriginal Ways of Learning and Learning Styles, paper presented at the Annual Conference of the Australian Association for Research in Education, Brisbane.

Johnson, S. (2001). Emergence: The connected lives of ants, brains, cities and software, New York: Scribner.

Ma Rhea, Z. (2004). The preservation and maintenance of the knowledge of Indigenous peoples and local communities: the role of education, Journal Of Australian Indigenous Issues, 7(1), 3-18.

Moore, R. and Young, M. (2001). Knowledge and the Curriculum in the Sociology of Education: Towards a Reconceptualisation, British Journal of Sociology of Education, 22(4), 445-461.

Penrose, R. (2004). The Road to Reality: A Complete Guide to the Laws of the Universe, Jonathon Cape.

Polkinghorne, D. E. (1988). Narrative Knowing and the Human Sciences, SUNY Press.

Reason, P. and Torbert, W. R. (2001). Toward a Transformational Science: A further look at the scientific merits of action research, Concepts and Transformations, 6(1), 1-37.

Sanderson, V. and Allard, A. (2001). 'Research as dialogue' and cross cultural consultations: confronting relations of power, paper presented at the Annual Conference of the Australian Association for Research in Education, Fremantle, December.

Schutz, A. (2001). John Dewey's Conundrum: Can Democratic Schools Empower? Teachers College Record, 103(2), 267-302. 
Schultz, K. (2001). Stretching The Boundaries Of Participatory Research: Insights From Conducting Research With Urban Adolescents, Australian Educational Researcher, 25(2), 1-28.

Shacklock, G. and Smyth, J. (1998). Being Reflexive in Critical Educational and Social Research, Falmer Press.

St Pierre, E. A. (2002). 'Science’ Rejects Postmodernism, Educational Researcher, 31(8).

Tricoglus, G. (2001). Living the Theoretical Principles of Critical Ethnography in Educational Research, Educational Action Research, 9(1), 143-146.

Young, R. (1990). A Critical Theory Of Education: Habermas and Our Children's Future, Teachers College Press. 
Figure 38.1. Emerging relationship between philosophy, education and organization

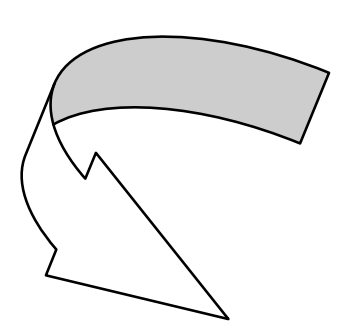

Philosophical Model

Critical Theory

Public Sphere

Indigenous Learning

Consciousness

Field, Habitus

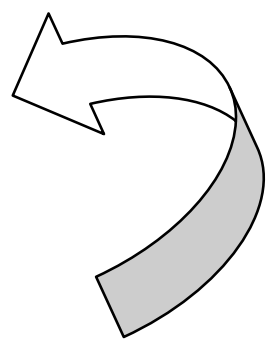

\section{Educational Concepts}

Learning Net

Democracy

Reflective unity of practice/theory

Inquiry, integrated learning

\section{Organisational Structure}

Spiral Curriculum

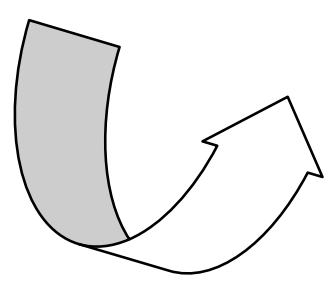

Narrative teams

Mentoring

Learning Circles 